\title{
Transmission dynamics of Monkeypox virus: a mathematical modelling approach
}

\author{
Olumuyiwa James Peter ${ }^{1}$ (1) Sumit Kumar ${ }^{2} \cdot$ Nitu Kumari $^{2} \cdot$ Festus Abiodun Oguntolu ${ }^{3} \cdot$ Kayode Oshinubi $^{4}$. \\ Rabiu Musa ${ }^{5}$
}

Received: 21 July 2021 / Accepted: 30 September 2021 / Published online: 15 October 2021

(c) The Author(s), under exclusive licence to Springer Nature Switzerland AG 2021

\begin{abstract}
Monkeypox (MPX), similar to both smallpox and cowpox, is caused by the monkeypox virus (MPXV). It occurs mostly in remote Central and West African communities, close to tropical rain forests. It is caused by the monkeypox virus in the Poxviridae family, which belongs to the genus Orthopoxvirus. We develop and analyse a deterministic mathematical model for the monkeypox virus. Both local and global asymptotic stability conditions for disease-free and endemic equilibria are determined. It is shown that the model undergo backward bifurcation, where the locally stable disease-free equilibrium coexists with an endemic equilibrium. Furthermore, we determine conditions under which the disease-free equilibrium of the model is globally asymptotically stable. Finally, numerical simulations to demonstrate our findings and brief discussions are provided. The findings indicate that isolation of infected individuals in the human population helps to reduce disease transmission.
\end{abstract}

Keywords Monkeypox virus $\cdot$ Mathematical model $\cdot$ Stability $\cdot$ Backward bifurcation

\section{Introduction}

Monkeypox is a severe viral zoonotic disease (i.e., animalto-human infection) that occurs sporadically, primarily in rural areas in Central and Western Africa, near tropical rainforests. This is caused by the monkeypox virus within the Poxviridae family that belongs to the genus Orthopoxvirus (Durski et al. 2018; Jezek et al. 1988). The genus Orthopoxvirus also comprises variola virus (the origin of smallpox), vaccinia virus (used for the eradication of smallpox in the vaccine), and cowpox virus (used in the earlier vaccine).

Olumuyiwa James Peter

peterjames4real@gmail.com

1 Department of Mathematics, University of Ilorin, Ilorin, Nigeria

2 School of Basic Sciences, Indian Institute of Technology Mandi, Mandi, Himachal Pradesh 175001, India

3 Department of Mathematics, Federal University of Technology Minna, Minna, Nigeria

4 AGIES Research Unit, Universite Grenoble Alpes, Alpes, France

5 School of Mathematics, Statistics and Computer Science, University of KwaZulu-Natal, Durban, South Africa
Monkeypox virus is mainly transmitted to humans from wild animals such as rodents and primates, but transmission often occurs from humans to humans. Human to human transmission has been linked to respiratory droplets and contact with bodily fluids, a contaminated patient's environment or items, and a skin lesion on an infected individual (Alakunle et al. 2020). Monkeypox virus has emerged as the most common orthopox virus after the eradication of the smallpox (Kantele et al. 2016). Fever, headache, muscle aches, backache, swollen lymph nodes, chills, and weariness are some of the symptoms that some individuals who may have contracted monkeypox experience. Up to a tenth of those infected with monkeypox die, with the majority of deaths happening in children under the age of ten (Nguyen et al. 2021).

Monkeypox was identified in 1958 when two pox-like disease outbreaks occurred in monk colonies held for study, hence the term 'monkeypox. The first human case was reported in the Democratic Republic of Congo in 1970 during a time of increased attempts to eradicate the smallpox. Among other Central and Western African countries like Cameroon, Gabon, Cote d'Ivoire, Liberia, Central African Republic, Congo, South Sudan and Sierra Leone, monkeypox has since been identified in humans. The first proof of 
monkeypox outbreaks in humans outside of Africa was a 2003 outbreak in the US. Monkeypox importation was later recognized in the United Kingdom and Israel. Mortality rate ranged from 1 percent to 10 percent in occurrences, with most deaths arising in younger populations (Ladnyj et al. 1972; CDC 2003). Monkeypox's incubation period is typically about $6-16$ days but can vary from 5 to 21 days. There are two facets of the contagious era, with an initial intrusive duration in the first 5 days, where the main signs are fever, lymphadenopathy (lymph node swelling), back pain, extreme headache, myalgia (muscle ache) and serious asthenia (energy shortage). A maculopapular rash (flatbased skin lesions) occurs 1-3 days after the onset of fever, and grows into small fluid-filled blisters (vesicles), which are pus-filled and then crust over in about ten days (Hutson et al. 2013).

Presently, there are no clear treatments available for monkeypox infection, though numerous novel antivirals, such as Brincindofovir, Tecovirimat and vaccinia immue globulin can be used to control the spread of the disease. There has been a significant increase in monkeypox in the last decade, associated with the decrease in herd immunity to smallpox. Vaccination against smallpox has been shown to be successful at 85 percent in the prevention of monkeypox but is no longer regularly available since global eradication of smallpox. The post-exposure vaccine can help prevent or decrease the severity of the disease (Rimoin et al. 2010; Meyer et al. 2020).

The disease has been given little attention in the past and this has contributed to insufficient knowledge on its mechanisms of transmission. Nevertheless, few studies have tried to research dynamics of monkeypox virus using a mathematical modelling technique. Study in Bhunu and Mushayabasa (2011) provides the basis for transmission analysis of pox-like dynamics of monkeypox virus as a case study. In Bhunu et al. (2009), the authors have shown that with the planned treatment intervention, the disease will be eradicated from both human and non-human primates in due time. The dynamics of monkeypox virus in human host and rodent with the stability analysis is studied in Usman and Adamu (2017). Other significant contributions can be found in TeWinkel (2019), Somma et al. (2019), Bankuru et al. (2020), Grant et al. (2020). Having gone through several works on the monkeypox virus and its mechanisms of transmission, we found that none considered the combination of isolated, exposed compartments in the human subpopulation and the effects of that contact rate with rodent population. Our aim is to investigate the various factors that could lead to reduction in the disease transmission and the effects of such factors on the basic reproduction number.

The rest of this paper is structured as follows: Method which includes model formulation and analysis are described in "Method" section. Next, "Backward bifurcation" section consists of the numerical simulations and results, discussion of results is given in "Results", "Discussion" sections. Finally, in "Conclusion" section, we have provided conclusions of this article. Table 1 shows a detailed description of the parameters, while the model's compartmental flow diagram is shown in Fig. 1.

\section{Method}

We propose a deterministic compartmental model on the transmission dynamics of monkeypox consisting of two populations that is, humans and rodents. The human population is further subdivided into five compartments, susceptible humans $S_{\mathrm{h}}(t)$, exposed humans $E_{\mathrm{h}}(t)$, infected humans $I_{\mathrm{h}}(t)$, isolated humans $Q_{\mathrm{h}}(t)$ and recovered humans $R_{\mathrm{h}}(t)$. The rodent population is subdivided into three compartments, susceptible rodents $S_{\mathrm{r}}(t)$, exposed rodents $E_{\mathrm{r}}(t)$ and infected rodents $I_{\mathrm{r}}(t)$. Recruitment into human population is at a rate $\theta_{\mathrm{h}} . \beta_{1}$ is the effective contact rate with the probability of human been infected with the virus per contact with an infected rodent and $\beta_{2}$ is the product of effective contact rate and the probability of human been infected with monkey pox virus after getting in contact with infectious human. The proportion of exposed individuals moving to highly infected class is $\alpha_{2}$ while the proportion identified is $\alpha_{1}$. After medical diagnosis, some suspected cases are confirmed, where others were not detected and returned back to susceptible humans a rate $\varphi$. The suspected cases are treated and moved to recovered class at a rate $\tau$. The recovery rate for human is at a rate $\gamma$. Natural death occurs in the humans and rodents population at the rates $\mu_{\mathrm{h}}$ and $\mu_{\mathrm{r}}$ respectively. $\beta_{3}$ is the effective contact rate with the probability of rodent been infected per contact with infected rodent. The infected rodent population decreased by natural mortality rate $\mu_{\mathrm{r}}$ or by disease induced death rate $\delta_{r}$. The transition among various compartments considered in the model is illustrated in Fig. 1, the model is governed by the following set of nonlinear differential equations below: 
Fig. 1 Schematic representation of the model

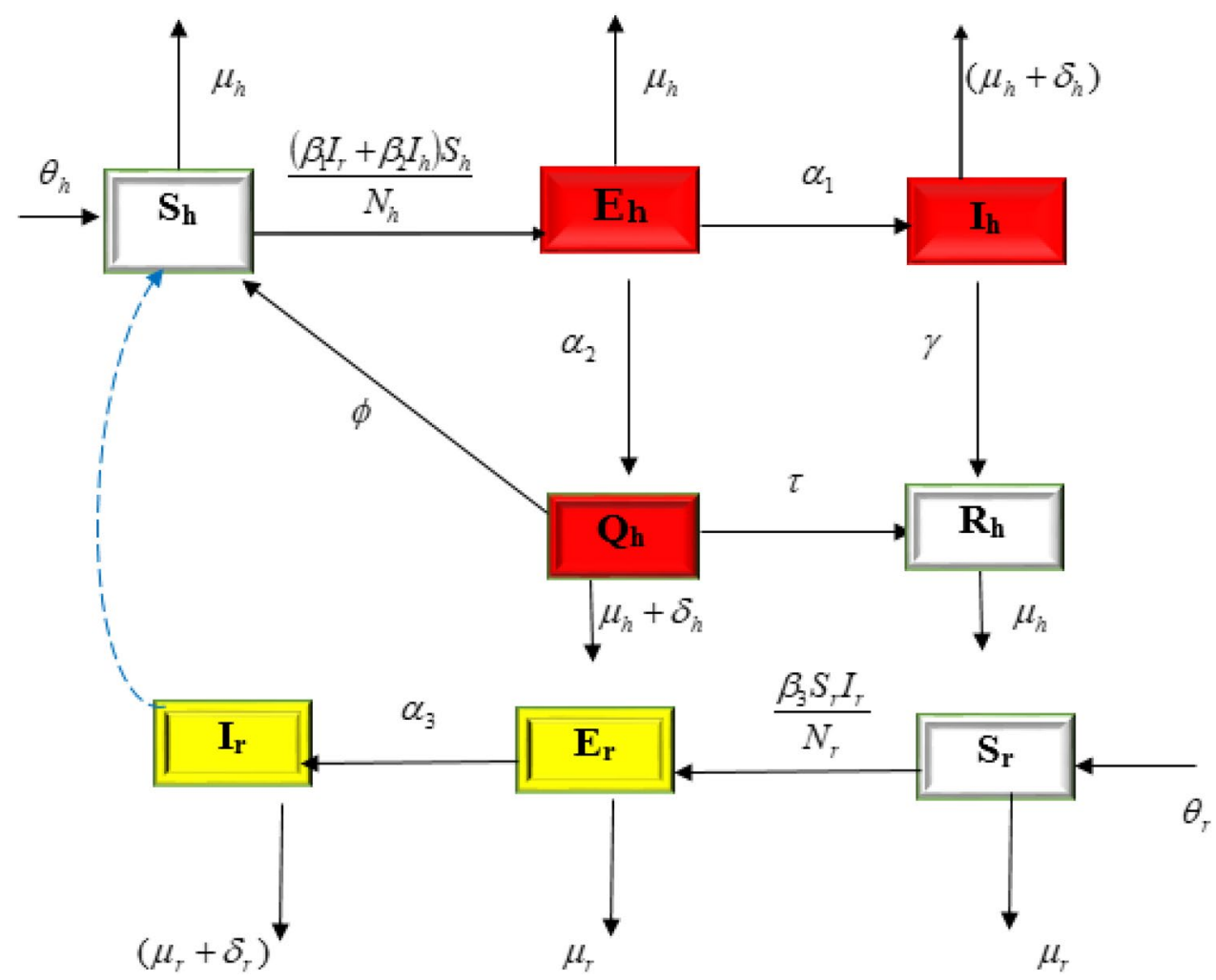

Table 1 Parameter values used for the simulations

\begin{tabular}{llll}
\hline Parameter & Value, Year $^{-1}$ & Source & Description \\
\hline$\theta_{\mathrm{h}}$ & 0.029 & Bhunu et al. (2009) & Recruitment rate for humans \\
$\theta_{\mathrm{r}}$ & 0.2 & Bhunu et al. (2009) & Recruitment rate for rodents \\
$\beta_{1}$ & 0.00025 & Bhunu and Mushayabasa (2011) & Rodent contact rate to humans \\
$\beta_{2}$ & 0.00006 & Bhunu and Mushayabasa (2011) & Human to humans contact rate \\
$\beta_{3}$ & 0.027 & Bhunu and Mushayabasa (2011) & Rodent to rodent contact rate \\
$\alpha_{1}$ & 0.2 & Assumed & Proportion of exposed human to infected humans \\
$\alpha_{2}$ & 2.0 & Estimated & Proportion identified as suspected case \\
$\varphi$ & 2.0 & Estimated & Proportion not detected after diagnosis \\
$\tau$ & 0.52 & Assumed & Progression from isolated to recovered class \\
$\gamma$ & 0.83 & Bhunu et al. (2009) & Humans recovery rate \\
$\mu_{\mathrm{h}}$ & 1.5 & Bhunu and Mushayabasa (2011) & Natural death rate of human \\
$\mu_{\mathrm{r}}$ & 0.002 & Bhunu and Mushayabasa (2011) & Natural death rate of rodents \\
$\delta_{\mathrm{r}}$ & 0.5 & Assumed & Disease induced death rate for rodents \\
$\delta_{\mathrm{h}}$ & 0.2 & Odom et al. (2009) & Disease induced death rate for humans \\
\hline
\end{tabular}


$\frac{\mathrm{d} S_{\mathrm{h}}}{\mathrm{d} t}=\theta_{\mathrm{h}}-\frac{\left(\beta_{1} I_{\mathrm{r}}+\beta_{2} I_{\mathrm{h}}\right) S_{\mathrm{h}}}{N_{\mathrm{h}}}-\mu_{\mathrm{h}} S_{\mathrm{h}}+\varphi Q_{\mathrm{h}}$

$\frac{\mathrm{d} E_{\mathrm{h}}}{\mathrm{d} t}=\frac{\left(\beta_{1} I_{\mathrm{r}}+\beta_{2} I_{\mathrm{h}}\right) S_{\mathrm{h}}}{N_{\mathrm{h}}}-\left(\alpha_{1}+\alpha_{2}+\mu_{\mathrm{h}}\right) E_{\mathrm{h}}$

$\frac{\mathrm{d} I_{\mathrm{h}}}{\mathrm{d} t}=\alpha_{1} E_{\mathrm{h}}-\left(\mu_{\mathrm{h}}+\delta_{\mathrm{h}}+\gamma\right) I_{\mathrm{h}}$

$\frac{\mathrm{d} Q_{\mathrm{h}}}{\mathrm{d} t}=\alpha_{2} E_{\mathrm{h}}-\left(\varphi+\tau+\delta_{\mathrm{h}}+\mu_{\mathrm{h}}\right) Q_{\mathrm{h}}$

$\frac{\mathrm{d} R_{\mathrm{h}}}{\mathrm{d} t}=\gamma I_{\mathrm{h}}+\tau Q_{\mathrm{h}}-\mu_{\mathrm{h}} R_{\mathrm{h}}$

$\frac{\mathrm{d} S_{\mathrm{r}}}{\mathrm{d} t}=\theta_{\mathrm{r}}-\frac{\beta_{3} S_{\mathrm{r}} I_{\mathrm{r}}}{N_{\mathrm{r}}}-\mu_{\mathrm{r}} S_{\mathrm{r}}$

$\frac{\mathrm{d} E_{\mathrm{r}}}{\mathrm{d} t}=\frac{\beta_{3} S_{\mathrm{r}} I_{\mathrm{r}}}{N_{\mathrm{r}}}-\left(\mu_{\mathrm{r}}+\alpha_{3}\right) E_{\mathrm{r}}$

$\frac{\mathrm{d} I_{\mathrm{r}}}{\mathrm{d} t}=\alpha_{3} E_{\mathrm{r}}-\left(\mu_{\mathrm{r}}+\delta_{\mathrm{r}}\right) I_{\mathrm{r}}$

\section{The model analysis}

For the human population, $N_{\mathrm{h}}=S_{\mathrm{h}}+E_{\mathrm{h}}+I_{\mathrm{h}}+Q_{\mathrm{h}}+R_{\mathrm{h}}$, the differential equation is given as:

$\left(\mathrm{d} N_{\mathrm{h}}\right) / \mathrm{d} t=\theta_{\mathrm{h}}-\delta_{\mathrm{h}} I_{\mathrm{h}}-\mu_{\mathrm{h}} N_{\mathrm{h}}$

Also, for the rodent population

$N_{\mathrm{r}}=S_{\mathrm{r}}+E_{\mathrm{r}}+I_{\mathrm{r}}$, and the corresponding differential equations is given as:

$\left(\mathrm{d} N_{\mathrm{r}}\right) / \mathrm{d} t=\theta_{\mathrm{r}}-\left(\mu_{\mathrm{r}}+\theta_{\mathrm{r}}\right) N_{\mathrm{r}}$

Theorem 1 Let $\left(S_{\mathrm{h}}, E_{\mathrm{h}}, I_{\mathrm{h}}, Q_{\mathrm{h}}, R_{\mathrm{h}}, S_{\mathrm{r}}, E_{\mathrm{r}}, R\right)$ be the solution of 1 with the initial conditions in a biologically feasible region $\Gamma=\Gamma_{\mathrm{h}} \times \Gamma_{\mathrm{r}}$ with:

$\Gamma_{\mathrm{h}}=S_{\mathrm{h}}, E_{\mathrm{h}}, I_{\mathrm{h}}, Q_{\mathrm{h}}, R_{\mathrm{h}} \in R_{+}^{5}: N_{\mathrm{h}} \leq \frac{\theta_{\mathrm{h}}}{\mu_{\mathrm{h}}}$

and

$\Gamma_{\mathrm{r}}=S_{\mathrm{r}}, E_{\mathrm{r}}, R_{\mathrm{r}} \in R_{+}^{3}: N_{\mathrm{r}} \leq \frac{\theta_{\mathrm{r}}}{\mu_{\mathrm{r}}}$

Then $\Gamma$ is non-negative invariant

Following the approach of Somma et al. (2019), we have that:

$0 \leq N_{\mathrm{h}}(t) \leq N_{\mathrm{h}}(0) \ell^{-\mu_{\mathrm{h}}(t)}+\frac{\theta_{\mathrm{h}}}{\mu_{\mathrm{r}}}\left(1-\ell^{-\mu_{\mathrm{h}}(t)}\right)$

also
$N_{\mathrm{r}}(t) \leq N_{\mathrm{r}}(0) \ell^{-\left(\mu_{\mathrm{r}}+\theta\right) t}+\frac{\theta_{\mathrm{r}}}{\mu_{\mathrm{r}}}\left(1-\ell^{-\left(\mu_{\mathrm{r}}+\theta\right) t}\right)$

Hence, the set $\Gamma$ is positive invariant and for $t$.

\section{Monkeypox-free equilibrium state}

This occurs in the absence of disease. Thus, in the absence of infection, we set $E_{\mathrm{h}}, I_{\mathrm{h}}, Q_{\mathrm{h}}, R_{\mathrm{h}}, E_{\mathrm{r}}$ and $I_{\mathrm{r}}$ to zero in 1 and the resulting solution gives the monkeypox-free equilibrium states given as:

$\Phi_{\mathrm{MFE}}\left({S_{\mathrm{h}}}^{*}, E_{\mathrm{h}}{ }^{*}, I_{\mathrm{h}}{ }^{*}, Q_{\mathrm{h}}{ }^{*}, R_{\mathrm{h}}{ }^{*}, S_{\mathrm{r}}{ }^{*} E_{\mathrm{r}}{ }^{*}, I_{\mathrm{r}}{ }^{*}\right)$

\section{Endemic equilibrium}

This occurs when the infection persist in the population represented by $\Phi_{\text {MEE }}\left(S^{*}{ }_{\mathrm{h}}, E^{*}{ }_{\mathrm{h}}, I^{*}{ }_{\mathrm{h}}, Q^{*}{ }_{\mathrm{h}}, R^{*}{ }_{\mathrm{h}}, S^{*}{ }_{\mathrm{r}}, E^{*}{ }_{\mathrm{r}}, I^{*}{ }_{\mathrm{r}}\right)$. Thus,

$$
\begin{aligned}
S_{\mathrm{h}}^{*} & =\frac{k_{1} k_{3} \theta_{\mathrm{h}}}{\mu_{\mathrm{h}} k_{1} k_{3}-\alpha_{2} \varphi \phi_{\mathrm{h}}+k_{1} k_{3} \phi_{\mathrm{h}}} \\
E^{*}{ }_{\mathrm{h}} & =\frac{k_{3} \phi_{\mathrm{h}} \theta_{\mathrm{h}}}{\mu_{\mathrm{h}} k_{1} k_{3}-\alpha_{2} \varphi \phi_{\mathrm{h}}+k_{1} k_{3} \phi_{\mathrm{h}}} \\
I^{*}{ }_{\mathrm{h}} & =\frac{k_{3} \alpha_{1} \phi_{\mathrm{h}} \theta_{\mathrm{h}}}{k_{2}\left(\mu_{\mathrm{h}} k_{1} k_{3}-\alpha_{2} \varphi \phi_{\mathrm{h}}+k_{1} k_{3} \phi_{\mathrm{h}}\right)} \\
Q_{\mathrm{h}}^{*} & =\frac{\alpha_{2} \phi_{\mathrm{h}} \theta_{\mathrm{h}}}{\mu_{\mathrm{h}} k_{1} k_{3}-\alpha_{2} \varphi \phi_{\mathrm{h}}+k_{1} k_{3} \phi_{\mathrm{h}}} \\
R_{\mathrm{h}}^{*} & =\frac{\left(\alpha_{1} \gamma k_{3}+\alpha_{2} k_{2} \tau\right) \phi_{\mathrm{h}} \theta_{\mathrm{h}}}{\mu_{\mathrm{h}} k_{2}\left(\mu_{\mathrm{h}} k_{1} k_{3}-\alpha_{2} \varphi \phi_{\mathrm{h}}+k_{1} k_{3} \phi_{\mathrm{h}}\right)} \\
S_{\mathrm{r}}^{*} & =\frac{\theta_{\mathrm{r}}}{\mu_{\mathrm{r}}+\phi_{\mathrm{r}}} \\
E_{\mathrm{r}}^{*} & =\frac{\theta_{\mathrm{r}}}{k_{4}\left(\mu_{\mathrm{r}}+\phi_{\mathrm{r}}\right)} \\
I_{\mathrm{r}}^{*} & =\frac{\phi_{\mathrm{r}} \alpha_{3} \theta_{\mathrm{r}}}{k_{4} k_{5}\left(\mu_{\mathrm{r}}+\phi_{\mathrm{r}}\right)}
\end{aligned}
$$

w h e r e $\quad k_{1}=\alpha_{1}+\alpha_{2}+\mu_{\mathrm{h}}, \quad k_{2}=\mu_{\mathrm{h}}+\delta_{\mathrm{h}}+\gamma$, $k_{3}=\varphi+\tau+\delta_{\mathrm{h}}+\mu_{\mathrm{h}}, \quad k_{4}=\mu_{\mathrm{r}}+\alpha_{3}, \quad k_{5}=\mu_{\mathrm{r}}+\delta_{\mathrm{r}}$, $\phi_{\mathrm{h}}=\frac{\beta_{1} I^{*}{ }_{\mathrm{r}}+\beta_{2} I_{\mathrm{h}}^{*}}{N_{\mathrm{h}}}, \phi_{\mathrm{r}}=\frac{\beta_{3} I^{*} \mathrm{r}}{N_{\mathrm{r}}}$.

\section{Basic reproduction number}

In our proposed model 1, compartments $S_{\mathrm{h}}, R_{\mathrm{h}}$ and $S_{\mathrm{r}}$ are the disease free states whereas the compartments $E_{\mathrm{h}}, I_{\mathrm{h}}, Q_{\mathrm{h}}, E_{\mathrm{r}}$ and $I_{\mathrm{r}}$ are the infection class.

Hence the monkeypox-free equilibrium state can be given as:

$\Phi_{\mathrm{MFE}}=\left(\frac{\theta_{\mathrm{h}}}{\mu_{\mathrm{h}}}, 0,0,0,0, \frac{\theta_{\mathrm{r}}}{\mu_{\mathrm{r}}}, 0,0\right)$ 
The basic reproduction number is one of the critical parameters to examine the long-term behaviour of an epidemic. It can be defined as the number of secondary cases produced by a single infected individual in its entire life span as infectious agent. We have used next-generation matrix technique explained in Diekmann et al. (2010), Peter et al. (2020), to obtain the expression of reproduction number $R_{0}$. It was first introduced by Driessche and Watmough van den Driessche and Watmough (2008), where this technique is discussed in detail for the estimation of $R_{0}$. Also, there are various articles available in literature where the next-generation matrix technique has been used to estimate the expression for the basic reproduction number (Samui et al. 2020; Kumar et al. 2021).

The model system 1 can be written as:

$$
\begin{aligned}
& \frac{\mathrm{d} x}{\mathrm{~d} t}=\mathcal{F}(x)-\mathcal{V}(x) \\
& \mathcal{F}=\left(\begin{array}{c}
\left(\beta_{1} I_{\mathrm{r}}+\beta_{2} I_{\mathrm{h}}\right. \\
N_{\mathrm{h}} \\
0 \\
0 \\
0 \\
0 \\
0 \\
0
\end{array}\right) S_{\mathrm{h}} \\
& \mathcal{V}=\left(\begin{array}{c}
-\theta_{\mathrm{h}}+\frac{\left(\beta_{1} I_{\mathrm{r}}+\beta_{2} I_{\mathrm{h}}\right) S_{\mathrm{h}}}{N_{\mathrm{h}}}+\mu_{\mathrm{h}} S_{\mathrm{h}}-\varphi Q_{\mathrm{h}} \\
\left(\alpha_{1}+\alpha_{2}+\mu_{\mathrm{h}}\right) E_{\mathrm{h}} \\
-\alpha_{1} E_{\mathrm{h}}+\left(\mu_{\mathrm{h}}+\delta_{\mathrm{h}}+\gamma\right) I_{\mathrm{h}} \\
-\alpha_{2} E_{\mathrm{h}}+\left(\varphi+\tau+\delta_{\mathrm{h}}+\mu_{\mathrm{h}}\right) Q_{\mathrm{h}} \\
-\gamma I_{\mathrm{h}}-\tau Q_{\mathrm{h}}+\mu_{\mathrm{h}} R_{\mathrm{h}} \\
-\theta_{\mathrm{r}}+\frac{\beta_{3} S_{\mathrm{r}} I_{\mathrm{r}}}{N_{\mathrm{r}}}+\mu_{\mathrm{r}} S_{\mathrm{r}} \\
-\frac{\beta_{3} S_{\mathrm{r}} I_{\mathrm{r}}}{N_{\mathrm{r}}}+\left(\mu_{\mathrm{r}}+\alpha_{3}\right) E_{\mathrm{r}} \\
-\alpha_{3} E_{\mathrm{r}}+\left(\mu_{\mathrm{r}}+\delta_{\mathrm{r}}\right) I_{\mathrm{r}}
\end{array}\right)
\end{aligned}
$$

Progression from $E_{\mathrm{h}}$ to $I_{\mathrm{h}}$ or $Q_{\mathrm{h}}$ are not considered to be new infections, but rather the progression of infected individuals through various compartments. Hence, the transmissions matrix $F$ and transitions matrix $V$ can be given as :

$F=\left(\begin{array}{cccc}0 & \beta_{2} & 0 & \beta_{1} \\ 0 & 0 & 0 & 0 \\ 0 & 0 & 0 & 0 \\ 0 & 0 & 0 & 0\end{array}\right)$

$V=\left(\begin{array}{cccc}\alpha_{1}+\alpha_{2}+\mu_{\mathrm{h}} & 0 & 0 & 0 \\ -\alpha_{1} & \mu_{\mathrm{h}}+\delta_{\mathrm{h}}+\gamma & 0 & 0 \\ -\alpha_{2} & 0 & \varphi \tau+\delta_{\mathrm{h}}+\mu_{\mathrm{h}} & 0 \\ 0 & 0 & 0 & \mu_{\mathrm{r}}+\delta_{\mathrm{r}}\end{array}\right)$

For simplicity, let $\Upsilon_{1}=\alpha_{1}+\alpha_{2}+\mu_{\mathrm{h}}, \Upsilon_{2}=\mu_{\mathrm{h}}+\delta_{\mathrm{h}}+\gamma, \Upsilon_{3}$ $=\varphi \tau+\delta_{\mathrm{h}}+\mu_{\mathrm{h}}$ and $\Upsilon_{4}=\mu_{\mathrm{r}}+\delta_{\mathrm{r}}$

Now:

$$
V^{-1}=\frac{1}{\Upsilon_{1} \Upsilon_{2} \Upsilon_{3} \Upsilon_{4}}\left(\begin{array}{cccc}
\Upsilon_{2} \Upsilon_{3} \Upsilon_{4} & 0 & 0 & 0 \\
\alpha_{1} \Upsilon_{3} \Upsilon_{4} & \Upsilon_{1} \Upsilon_{3} \Upsilon_{4} & 0 & 0 \\
\alpha_{2} \Upsilon_{2} \Upsilon_{4} & 0 & \Upsilon_{1} \Upsilon_{2} \Upsilon_{4} & 0 \\
0 & 0 & 0 & \Upsilon_{1} \Upsilon_{2} \Upsilon_{3}
\end{array}\right)
$$

Now, after much simplification we obtain:

$$
\mathrm{FV}^{-1}=\frac{1}{\Upsilon_{1} \Upsilon_{2} \Upsilon_{3} \Upsilon_{4}}\left(\begin{array}{cccc}
\beta_{2} \alpha_{1} \Upsilon_{3} \Upsilon_{4} & 0 & 0 & \beta_{1} \Upsilon_{1} \Upsilon_{2} \Upsilon_{3} \\
0 & 0 & 0 & 0 \\
0 & 0 & 0 & 0 \\
0 & 0 & 0 & 0
\end{array}\right)
$$

Now, the basic reproduction number is defined as the largest eigenvalue (spectral radius) of the next generation matrix $\mathrm{FV}^{-1}$ and can be obtained as:

$R_{0}=\rho\left(\mathrm{FV}^{-1}\right)=\frac{\beta_{2} \alpha_{1} \Upsilon_{3} \Upsilon_{4}}{\Upsilon_{1} \Upsilon_{2} \Upsilon_{3} \Upsilon_{4}}=\frac{\beta_{2} \alpha_{1}}{\Upsilon_{1} \Upsilon_{2}}$

Hence,

$R_{0}=\frac{\alpha_{1} \beta_{2}}{\left(\alpha_{1}+\alpha_{2}+\mu_{\mathrm{h}}\right)\left(\mu_{\mathrm{h}}+\delta_{\mathrm{h}}+\gamma\right)}$

\section{Stability of disease-free equilibrium}

To obtain the conditions for the global stability for $E_{0}$, we have used the approach set out in Castillo-Chavez and Song (2004), which states that if the model system can be written in the following form:

$\frac{\mathrm{d} X}{\mathrm{~d} t}=F(X, Z)$

$\frac{\mathrm{d} Z}{\mathrm{~d} t}=G(X, Z), G(X, 0)=0$

here $X \in R^{n}$ are the uninfected individuals and $Z \in R^{m}$ describes the infected individuals. According to this notation, the disease-free equilibrium is given by $Q_{0}=\left(X_{0}, 0\right)$. Now, the following two conditions guarantees the global stability of the disease free equilibrium.

K1 : For $\frac{\mathrm{d} X}{\mathrm{~d} t}=F(X, 0), X_{0}$ is globally asymptotically stable. $\mathrm{K} 2: \quad G(X, Z)=B Z-\hat{G}(X, Z)$ where $\hat{G}(X, Z) \geq 0$ for $X, Z \in \Omega$.

here $B=D_{z} G\left(X_{0}, 0\right)$ is a $M$-matrix and $\Omega$ is the feasible of the model. The following theorem then defines the global stability of $E_{0}$.

Lemma 1 The equilibrium point $Q_{0}=\left(X_{0}, 0\right)$ is a globally asymptotically stable when $R_{0} \leq 1$ and assumptions $K_{1}$ and $\mathrm{K}_{2}$ are satisfied. 
Now, the following theorem establishes the global stability of the disease free equilibrium $E_{0}$ for our proposed model system.

Theorem 2 The DFE point $E_{0}$ is globally asymptotically stable provided $R_{0} \leq 1$.

Proof First, we will prove $K_{1}$ as:

$F(X, 0)=\left[\begin{array}{c}\theta_{\mathrm{h}}-\mu_{\mathrm{h}} S_{\mathrm{h}} \\ -\mu_{\mathrm{h}} R_{\mathrm{h}} \\ \theta_{\mathrm{r}}-\mu_{\mathrm{r}} S_{\mathrm{r}} \\ -\left(\mu_{\mathrm{r}}+\alpha_{3}\right) E_{\mathrm{r}}\end{array}\right]$

The characteristic polynomial of $F(X, 0)$ is:

$\left(\lambda+\mu_{\mathrm{h}}\right)^{2}\left(\lambda+\mu_{\mathrm{r}}\right)\left(\lambda+\mu_{\mathrm{r}}+\alpha_{3}\right)$

$\Rightarrow \lambda_{1}=\lambda_{2}=-\mu_{\mathrm{h}}, \lambda_{3}=-\mu_{\mathrm{r}}$ and $\lambda_{4}=-\mu_{\mathrm{r}}-\alpha_{3}$.

Hence, $X=X_{0}$ is globally asymptotically stable.

Now, we have:

$G(X, Z)=B Z-\hat{G}(X, Z)$

$=\left[\begin{array}{cccc}-\left(\alpha_{1}+\alpha_{2}+\mu_{\mathrm{h}}\right) & \frac{\beta_{2} S_{\mathrm{h}}^{0}}{N_{\mathrm{h}}} & 0 & \frac{\beta_{1} S_{\mathrm{h}}^{0}}{N_{\mathrm{h}}} \\ \alpha_{1} & -\left(\mu_{\mathrm{h}}+\delta_{\mathrm{h}}+\gamma\right) & 0 & 0 \\ \alpha_{2} & 0 & -\left(\varphi+\tau+\delta_{\mathrm{h}}+\mu_{\mathrm{h}}\right) & 0 \\ 0 & 0 & 0 & \mu_{\mathrm{r}}+\delta_{\mathrm{r}}\end{array}\right]$ $\times\left[\begin{array}{c}E_{\mathrm{h}} \\ I_{\mathrm{h}} \\ Q_{\mathrm{h}} \\ I_{\mathrm{r}}\end{array}\right]-\left[\begin{array}{c}\left(\frac{\beta_{2}\left(S_{\mathrm{h}}^{0}-S_{\mathrm{h}}\right)+\beta_{1}\left(S_{\mathrm{h}}^{0}-S_{\mathrm{h}}\right)}{N_{\mathrm{h}}}\right) E_{\mathrm{h}} \\ 0 \\ 0 \\ \alpha_{3} E_{\mathrm{r}}\end{array}\right]$

Here, one can easily observe that $B$ satisfies all conditions explained in $\mathrm{K} 2$.

\section{Stability of endemic equilibrium}

We will use the Routh-Hurwitz criterion to prove the local stability of the endemic equilibria. Here, we will derive the conditions under which the endemic equilibria is locally asymptotically stable.

The Jacobian matrix about the endemic equilibria $\phi_{\mathrm{MEE}}$ is given as :

$$
J=\left[\begin{array}{cccccccc}
a_{11} & 0 & a_{13} & a_{14} & 0 & 0 & 0 & a_{18} \\
a_{21} & a_{22} & a_{23} & 0 & 0 & 0 & 0 & a_{28} \\
0 & a_{32} & a_{33} & 0 & 0 & 0 & 0 & 0 \\
0 & a_{42} & 0 & a_{44} & 0 & 0 & 0 & 0 \\
0 & 0 & a_{53} & a_{54} & a_{55} & 0 & 0 & 0 \\
0 & 0 & 0 & 0 & 0 & a_{66} & 0 & a_{68} \\
0 & 0 & 0 & 0 & 0 & a_{76} & a_{77} & a_{78} \\
0 & 0 & 0 & 0 & 0 & 0 & a_{87} & a_{88}
\end{array}\right]
$$

Here,

$a_{11}=-\left(\frac{\beta_{1} I_{\mathrm{r}}+\beta_{2} I_{\mathrm{h}}}{N_{\mathrm{h}}}\right)-\mu_{\mathrm{h}} \quad a_{13}=-\frac{\beta_{2} S_{\mathrm{h}}}{N_{\mathrm{h}}}$

$a_{14}=\phi \quad a_{18}=-\frac{\beta_{1} S_{\mathrm{h}}}{N_{\mathrm{h}}}$

$a_{21}=\frac{\beta_{1} I_{\mathrm{r}}+\beta_{2} I_{\mathrm{h}}}{N_{\mathrm{h}}} \quad a_{22}=-\left(\alpha_{1}+\alpha_{2}+\mu_{\mathrm{h}}\right)$

$a_{23}=\frac{\beta_{2} S_{\mathrm{h}}}{N_{\mathrm{h}}} \quad a_{28}=\frac{\beta_{1} S_{\mathrm{h}}}{N_{\mathrm{h}}}$

$a_{32}=\alpha_{1} \quad a_{33}=-\left(\mu_{\mathrm{h}}+\delta_{\mathrm{h}}+\gamma\right)$

$a_{42}=\alpha_{2} \quad a_{44}=-\left(\varphi+\tau+\delta_{\mathrm{h}}+\mu_{\mathrm{h}}\right)$

$a_{53}=\gamma \quad a_{54}=\tau \quad a_{55}=-\mu_{\mathrm{h}}$

$a_{66}=-\left(\mu_{\mathrm{r}}+\frac{\beta_{3} I_{\mathrm{r}}}{N_{\mathrm{r}}}\right) \quad a_{68}=-\frac{\beta_{3} S_{\mathrm{r}}}{N_{\mathrm{r}}}$

$a_{76}=\frac{\beta_{3} I_{\mathrm{r}}}{N_{\mathrm{r}}} \quad a_{77}=-\left(\mu_{\mathrm{r}}+\alpha_{3}\right)$

$a_{78}=\frac{\beta_{3} S_{\mathrm{r}}}{N_{\mathrm{r}}} \quad a_{87}=\alpha_{3} \quad a_{88}=-\left(\mu_{\mathrm{r}}+\delta_{\mathrm{r}}\right)$

The characteristic equation of $J$ is given as:

$$
\begin{aligned}
& \frac{1}{N_{\mathrm{h}} N_{\mathrm{r}}}\left[( - x - \mu _ { \mathrm { h } } ) \left(-\phi \alpha_{2}\left(I_{\mathrm{r}} \beta_{1}+I_{\mathrm{h}} \beta_{2}\right)\right.\right. \\
& \quad\left(x+\gamma+\delta_{\mathrm{h}}+\mu_{\mathrm{h}}\right)+\left(-x-\tau-\varphi-\delta_{\mathrm{h}}-\mu_{\mathrm{h}}\right) \\
& \quad\left(S_{\mathrm{h}} \alpha_{1} \beta_{2}\left(x+\mu_{\mathrm{h}}\right)-\left(x+\alpha_{1}+\alpha_{2}+\mu_{\mathrm{h}}\right)\right. \\
& \left.\left.\quad\left(x+\gamma+\delta_{\mathrm{h}}+\mu_{\mathrm{h}}\right)\left(I_{\mathrm{r}} \beta_{1}+I_{\mathrm{h}} \beta_{2}+N_{\mathrm{h}}\left(x+\mu_{\mathrm{h}}\right)\right)\right)\right) \\
& \left(S_{\mathrm{r}} \alpha_{3} \beta_{3}\left(x+\mu_{\mathrm{r}}\right)-\left(x+\alpha_{3}+\mu_{\mathrm{r}}\right)\right. \\
& \left.\left.\quad\left(I_{\mathrm{r}} \beta_{3}+N_{\mathrm{r}}\left(x+\mu_{\mathrm{r}}\right)\right)\left(x+\left(\mu_{\mathrm{r}}+\delta_{\mathrm{r}}\right)\right)\right)\right]=0
\end{aligned}
$$

which can be further written as:

$$
\begin{aligned}
x^{8} & +A_{1} x^{7}+A_{2} x^{6}+A_{3} x^{5}+A_{4} x^{4} \\
& +A_{5} x^{3}+A_{6} x^{2}+A_{7} x+A_{8}=0
\end{aligned}
$$

where $A_{i}$ 's are the coefficients of $x^{8-i} ; i=1,2, \ldots 8$ after converting the polynomial in standard form.

Note: To obtain the condition for the stability of $\phi_{\mathrm{MEE}}$ we will made the following substitution: 
$P=\frac{A_{1} A_{2}-A_{0} A_{3}}{A_{1}}, \quad Q=\frac{A_{1} A_{4}-A 0 A_{5}}{A_{1}}$,

$R=\frac{A_{1} A_{6}-A_{0} A_{7}}{A_{1}}, \quad S=A_{8}$,

$P^{*}=\frac{p A_{3}-A_{1} Q}{P}, \quad Q^{*}=\frac{P A_{5}-A_{1} R}{P}$,

$R^{*}=\frac{P A_{7}-A_{1} S}{P}, \quad M=\frac{P^{*} Q-P Q^{*}}{P^{*}}$,

$N=\frac{P^{*} R-P R^{*}}{P^{*}}, \quad T=\frac{P^{*} S}{P^{*}}$,

$M^{*}=\frac{M Q^{*}-P^{*} N}{M}, \quad N^{*}=\frac{M R^{*}-P^{*} T}{M}$,

$X=\frac{M^{*} N-M N^{*}}{M^{*}}$.

Hence, we can conclude this section by the following theorem:

Theorem 3 The endemic equilibrium point $\phi_{\mathrm{MEE}}$ is locally asymptotically stable provided $R_{0}>1$ and following conditions are satisfied:

$A_{1}>0$.

$A_{1} A_{2}>A_{3}$.

$A_{1} A_{2} A_{3}+A_{0} A_{1} A_{5}>A_{0} A_{3}^{2}+A_{1}^{2} A_{4}$

$P^{*} Q>P Q^{*} \quad M Q^{*}>P^{*} N \quad M^{*} N>M N^{*} \quad X N^{*}>T M^{*}$

\section{Backward bifurcation}

The analysis conducted in the previous section on the occurrence of endemic equilibrium $E^{*}$ suggests the probability of backward bifurcation. It can be defined as the state when a stable endemic equilibrium coexist with with a stable disease-free equilibrium when the associated reproduction number is less than unity. We use the center manifold based result (theorem 4.1) given in Castillo-Chavez and Song (2004), to check the occurrence of backward bifurcation.

Let:

$S_{\mathrm{h}}=y_{1}, \quad E_{\mathrm{h}}=y_{2}, \quad I_{\mathrm{h}}=y_{3}, \quad Q_{\mathrm{h}}=y_{4}$,

$R_{\mathrm{h}}=y_{5}, \quad S_{\mathrm{r}}=y_{6}, \quad E_{\mathrm{r}}=y_{7}, \quad I_{\mathrm{r}}=y_{8}$.

Consider, $U=\left(y_{1}, y_{2}, y_{3}, y_{4}, y_{5}, y_{6}, y_{7}, y_{8},\right)^{T}$, then the given system (1) can be written as:

$\frac{\mathrm{d} U}{\mathrm{~d} t}=\left(f_{1}, f_{2}, f_{3}, f_{4}, f_{5}, f_{6}, f_{7}, f_{8}\right)^{T}$ where,

$$
\begin{aligned}
& f_{1}=\theta_{\mathrm{h}}-\frac{\left(\beta_{1} y_{8}+\beta_{2} y_{3}\right) S_{\mathrm{h}}}{N_{\mathrm{h}}}-\mu_{\mathrm{h}} y_{1}+\phi y_{4} \\
& f_{2}=\frac{\left(\beta_{1} y_{8}+\beta_{2} y_{3}\right) S_{\mathrm{h}}}{N_{\mathrm{h}}}-\left(\alpha_{1}+\alpha_{2}+\mu_{\mathrm{h}}\right) y_{2} \\
& f_{3}=\alpha_{1} y_{2}-\left(\mu_{\mathrm{h}}+\delta_{\mathrm{h}}+\gamma\right) y_{3} \\
& f_{4}=\alpha_{2} y_{2}-\left(\varphi+\tau+\delta_{\mathrm{h}}+\mu_{\mathrm{h}}\right) y_{4} \\
& f_{5}=\gamma y_{3}+\tau y_{4}-\mu_{\mathrm{h}} y_{5} \\
& f_{6}=\theta_{\mathrm{r}}-\frac{\beta_{3} y_{6} y_{8}}{N_{\mathrm{r}}}-\mu_{\mathrm{r}} y_{6} \\
& f_{7}=\frac{\beta_{3} y_{6} y_{8}}{N_{\mathrm{r}}}-\left(\mu_{\mathrm{r}}+\alpha_{3}\right) y_{7} \\
& f_{8}=\alpha_{3} y_{7}-\left(\mu_{\mathrm{r}}+\delta_{\mathrm{r}}\right) y_{8}
\end{aligned}
$$

From the expression of $R_{0}$, we can observe that $R_{0}$ is highly influenced by $\beta_{2}$, the product of effective contact rate and the probability of human been infected with monkey pox virus after getting in contact with infectious human. Therefore, we will consider $\beta_{2}$ as our bifurcation parameter.

Hence, when $R_{0}=1$, we have:

$\beta_{2}^{*}=\frac{\left(\alpha_{1}+\alpha_{2}+\mu_{\mathrm{h}}\right)\left(\mu_{\mathrm{h}}+\delta_{\mathrm{h}}+\gamma\right)}{\alpha_{1}}$

Now, the above system at monkeypox-free equilibrium state $\phi_{\mathrm{MFE}}$ is given by:

$J_{0}\left(\phi_{\mathrm{MFE}}, \beta_{2}^{*}\right)=\left[\begin{array}{cccccccc}-\mu_{\mathrm{h}} & 0 & -\beta_{2} & 0 & 0 & 0 & 0 & -\beta_{1} \\ 0 & -A_{1} & \beta_{2} & 0 & 0 & 0 & 0 & \beta_{1} \\ 0 & \alpha_{1} & -A_{2} & 0 & 0 & 0 & 0 & 0 \\ 0 & \alpha_{2} & 0 & -A_{3} & 0 & 0 & 0 & 0 \\ 0 & 0 & \gamma & \tau & -\mu_{\mathrm{h}} & 0 & 0 & 0 \\ 0 & 0 & 0 & 0 & 0 & -\mu_{\mathrm{r}} & 0 & 0 \\ 0 & 0 & 0 & 0 & 0 & 0 & -A_{4} & \beta_{3} \\ 0 & 0 & 0 & 0 & 0 & 0 & \alpha_{3} & -A_{5}\end{array}\right]$

Clearly, ' 0 ' is an eigenvalue of $J_{0}\left(\phi_{\mathrm{MFE}}, \beta_{2}^{*}\right)$. Let $W=\left(w_{1}, w_{2}, w_{3}, w_{4}, w_{5}, w_{6}, w_{7}, w_{8}\right)$ be the associated right eigenvector corresponding to zero eigenvalue, and can be attained by simplifying:

$$
\begin{aligned}
& -\mu_{\mathrm{h}} w_{1}-\beta_{2} w_{3}-\beta_{1} w_{8}=0 \\
& -A_{1} w_{2}+\beta_{2} w_{3}+\beta_{1} w_{8}=0 \\
& \alpha_{1} w_{2}-A_{2} w_{3}=0 \\
& \alpha_{2} w_{2}-A_{3} w_{4}=0 \\
& \gamma w_{3}+\tau w_{4}-\mu_{\mathrm{h}} w_{5}=0 \\
& -\mu_{\mathrm{r}} w_{6}-\beta_{3} w_{8}=0 \\
& -A_{4} w_{7}+\beta_{3} w_{8}=0 \\
& \alpha_{3} w_{7}-A_{5} w_{8}=0
\end{aligned}
$$

On evaluation, $W$ can be given as: 
$w_{1}=-\frac{A_{1} A_{2}}{\alpha_{1} \mu_{\mathrm{h}}} \quad w_{2}=\frac{A_{2}}{\alpha_{1}} \quad w_{3}=1$

$w_{4}=\frac{\alpha_{2} A_{2}}{\alpha_{1} A_{3}} \quad w_{5}=\frac{1}{\mu_{\mathrm{h}}}\left(\gamma+\frac{\tau \alpha_{2} A_{2}}{\alpha_{1} A_{3}}\right)$

$w_{6}=\frac{\beta_{3}}{\beta_{1} \mu_{\mathrm{r}}}\left(\frac{A_{1} A_{2}}{\alpha_{1}}+\beta_{2}\right)$

$w_{7}=-\frac{A_{5}}{\alpha_{3} \beta_{1}}\left(\frac{A_{1} A_{2}}{\alpha_{1}}+\beta_{2}\right) \quad w_{8}=-\frac{1}{\beta_{1}}\left(\frac{A_{1} A_{2}}{\alpha_{1}}+\beta_{2}\right)$

Now, let $V=\left(v_{1}, v_{2}, v_{3}, v_{4}, v_{5}, v_{6}, v_{7}, v_{8}\right)$ be the associated left eigenvector of $J_{0}$ corresponding to zero eigenvalue and satisfying $V . W=0$. Then $V$ can be given as :

$v_{1}=0$,

$v_{2}=\left(\frac{A_{2}}{\alpha_{1}}+\frac{\beta_{2}}{A_{2}}-\left(\left(\frac{A_{1} A_{3}}{\alpha_{1}}+\beta_{2}\right)\right.\right.$

$\left.\cdot \frac{1}{\left(\frac{A_{4} A_{5}}{\alpha_{3}}-\beta_{3}\right)} \times\left(\frac{A_{5}}{\alpha_{3}}+\frac{\beta_{2}}{A_{2}}\right)\right)^{-1}$,

$v_{3}=\frac{\beta_{2}}{A_{2}} \cdot v_{2}, \quad v_{4}=v_{5}=v_{6}=0$,

$v_{7}=\frac{\beta_{1}}{\left(\frac{A_{4} A_{5}}{\alpha_{3}}-\beta_{3}\right)} \cdot v_{2}, \quad v_{8}=\frac{\beta_{2}}{A_{2}} \cdot v_{7}$

As discussed in theorem 4.1 (Castillo-Chavez and Song 2004), we have:

$a=\sum_{k, i, j=1}^{8} v_{k} w_{i} w_{j} \frac{\partial^{2} f_{k}}{\partial y_{i} \partial y_{j}}\left(\phi_{\mathrm{MFE}}, \beta_{2}^{*}\right)$

$b=\sum_{k, i=1}^{8} v_{k} w_{i} \frac{\partial^{2} f_{k}}{\partial y_{i} \partial \beta_{2}}\left(\phi_{\mathrm{MFE}}, \beta_{2}^{*}\right)$

Algebraic calculations shows that:

$$
\begin{aligned}
\frac{\partial^{2} f_{2}}{\partial x_{1} x_{3}} & =\frac{\beta_{2}}{N_{\mathrm{h}}}=\frac{\partial^{2} f_{2}}{\partial x_{3} x_{1}} \quad \frac{\partial^{2} f_{2}}{\partial x_{1} x_{8}}=\frac{\beta_{1}}{N_{\mathrm{h}}}=\frac{\partial^{2} f_{2}}{\partial x_{8} x_{1}} \\
\frac{\partial^{2} f_{7}}{\partial x_{8} x_{6}} & =\frac{1}{N_{\mathrm{r}}}=\frac{\partial^{2} f_{7}}{\partial x_{6} x_{8}}
\end{aligned}
$$

Now, substituting all the above values in the expressions for ' $a$ ' and ' $b$ ', we obtain:

$a=\frac{2 v_{2} w_{1}}{N_{\mathrm{h}}}\left(w_{3} \beta_{2}+w_{8} \beta_{1}\right)+2 v_{7} \frac{w_{6} w_{8}}{N_{\mathrm{r}}}$

$b=v_{2} \cdot w_{2} \cdot \frac{\theta}{\mu_{\mathrm{h}} N_{\mathrm{h}}}$
Now, to persist backward bifurcation in the proposed model, both the values of ' $a$ ' and ' $b$ ' has to be simultaneously positive.

\section{Results}

A sensitivity analysis determines how different values of an independent variable affect a particular dependent variable under a given set of assumptions (Kalyan et al. 2021; Victorr et al. 2020). The normalized forward sensitivity index of a variable to a parameter is the ratio of the relative change in the variable to the relative change in the parameter. When variable is a differentiable function of the parameter, the sensitivity index may be alternatively defined using partial derivatives. The parameter values have been taken from literature as given in Table 1.

Since the basic reproduction number $R_{0}$ helps us to predict the future course of the disease, the sensitivity analysis is performed to understand which parameters involved in the model effect the value of $R_{0}$ relatively more. We have used the following expression of the sensitivity for $R_{0}$ which depends upon parameter $v$.

$\psi_{v}^{R_{0}}=\frac{v}{R_{0}} \times \frac{\partial R_{0}}{\partial v}$

A negative index of sensitivity shows that the parameter and $R_{0}$ are inversely proportional. A positive sensitivity index, however, denotes that the value of $R_{0}$ increases with an increase in the value of the parameter concerned.

The estimated sensitivity indices for $R_{0}$ are presented in Table 2. From Table 2, we can see that an increase in the values of $\alpha_{2}, \mu_{\mathrm{h}}, \delta_{\mathrm{h}}$ and $\gamma$ will results in a decrease in the value of $R_{0}$. On the another hand, an increase in the value of $\alpha_{1}$ and $\beta_{2}$ will increase the monkey-pox cases.

Table 2 Sensitivity index of parameters

\begin{tabular}{lll}
\hline Parameter & $\begin{array}{l}\text { Expression of the sensitivity } \\
\text { index }\end{array}$ & Value \\
\hline$\alpha_{1}$ & $\frac{\alpha_{2}+\mu_{\mathrm{h}}}{\alpha_{1}+\alpha_{2}+\mu_{\mathrm{h}}}$ & 0.945946 \\
$\alpha_{2}$ & $-\frac{\alpha_{2}}{\alpha_{1}+\alpha_{2}+\mu_{\mathrm{h}}}$ & -0.540541 \\
$\beta_{2}$ & 1 & 1 \\
$\mu_{\mathrm{h}}$ & $-\frac{\mu_{\mathrm{h}}\left(\gamma+\alpha_{1}+\alpha_{2}+\delta_{\mathrm{h}}+2 \mu_{\mathrm{h}}\right)}{\left(\alpha_{1}+\alpha_{2}+\mu_{\mathrm{h}}\right)\left(\gamma+\delta_{\mathrm{h}}+\mu_{\mathrm{h}}\right)}$ & -0.998291 \\
$\delta_{\mathrm{h}}$ & $-\frac{\delta_{\mathrm{h}}}{\gamma+\delta_{\mathrm{h}}+\mu_{\mathrm{h}}}$ & -0.0790514 \\
$\gamma$ & $-\frac{\gamma}{\gamma+\delta_{\mathrm{h}}+\mu_{\mathrm{h}}}$ & -0.328063 \\
\hline
\end{tabular}




\section{Discussion}

The basic reproduction number is a crucial parameter in disease dynamics which gives us major information about the disease. To understand the effect of various disease transmission parameters on the basic reproduction number, we have obtained the surface plots showing variation of $R_{0}$ with sensitive parameters. From Fig. 2, it can be observed that as the value of $\alpha_{2}$ increases, it leads to reduced disease transmission. Similarly, it can be easily seen from Fig. 3 that contact rate with rodent population directly affects the transmission of monkey-pox. Similarly, the simultaneous effect of $\beta_{2}, \alpha_{2}, \mu_{\mathrm{h}}$ and $\gamma$ on the basic reproduction number has been shown in Figs. 4 and 5.

Further, we have performed numerical experiments to detect effect of change in sensitive parameters on the number of infected individuals. This has been investigated in Figs. 6, 7 and 8. Now we have incorporated a compartment
$Q_{\mathrm{h}}$ in the model, which consists of the isolated proportion of the infected humans. Through numerical simulations, we have shown how the infected population would behave in the absence of isolated interventions. In Fig. 9, we show that the isolation of infected individuals helps to reduce disease transmission.

\section{Conclusion}

A non-linear compartmental model has been proposed to understand the transmission of Monkey pox disease. The proposed model consist of eight mutually exclusive compartments. The human population has been divided into five compartments, where we has introduced the exposed $\left(E_{\mathrm{h}}\right)$ and isolated human $\left(Q_{\mathrm{h}}\right)$ compartments along with standard compartments of exposed population $\left(E_{\mathrm{h}}\right)$, infected humans $\left(I_{\mathrm{h}}\right)$ and recovered humans $\left(R_{\mathrm{h}}\right)$. Similarly, the rodent population is also divided into three compartments; exposed
Fig. 2 Surface plot showing simultaneous impact of $\alpha_{1}$ and $\alpha_{2}$ on $R_{0}$

Fig. 3 Surface plot showing simultaneous impact of $\alpha_{1}$ and $\beta_{2}$ on $R_{0}$
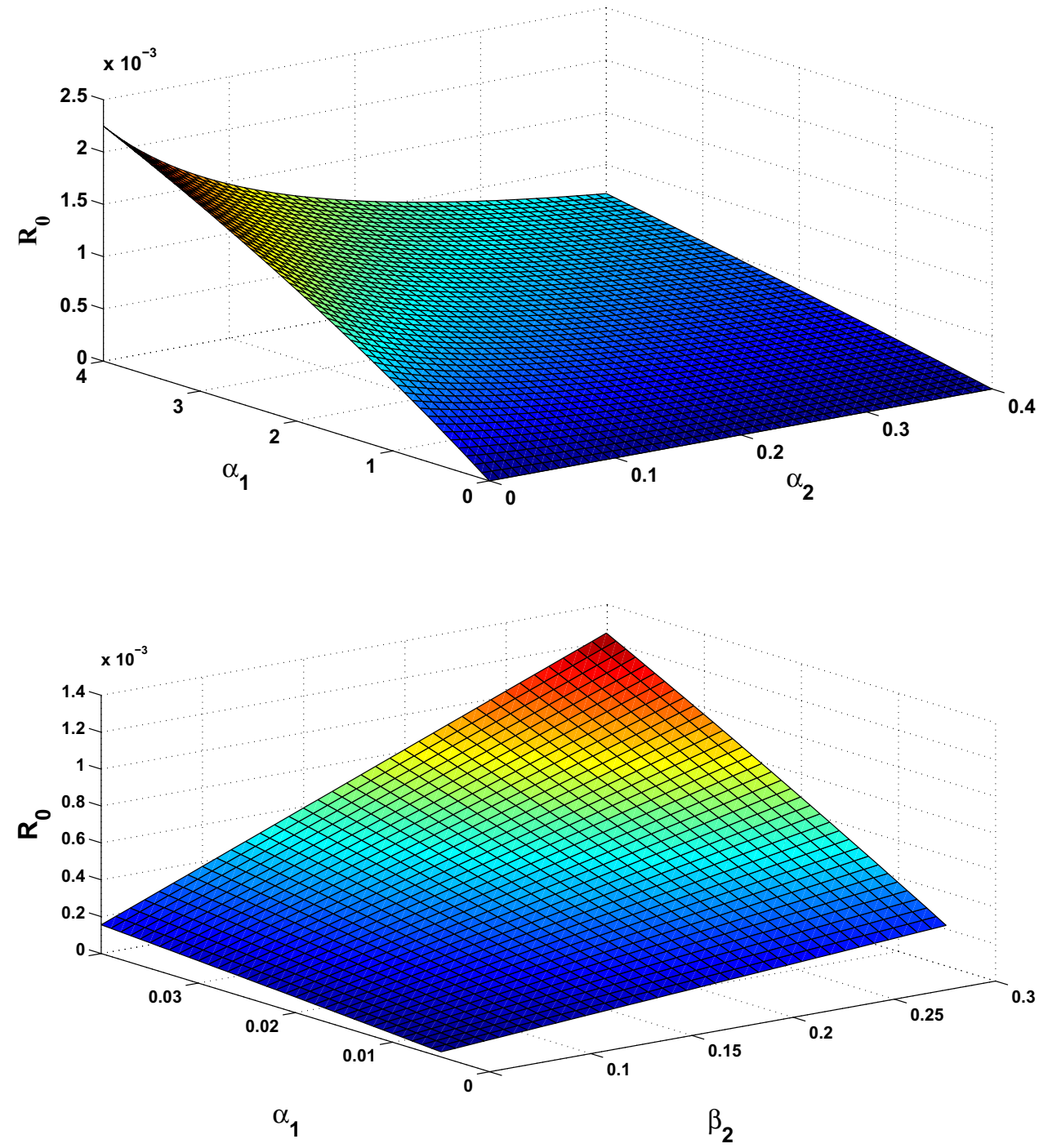
Fig. 4 Surface plot showing simultaneous impact of $\beta_{2}$ and $\alpha_{2}$ on $R_{0}$

Fig. 5 Surface plot showing simultaneous impact of $\mu_{\mathrm{h}}$ and $\gamma$ on $R_{0}$

Fig. 6 Variation in infected population over time for different values of $\alpha_{1}$; proportion of humans exposed to infection
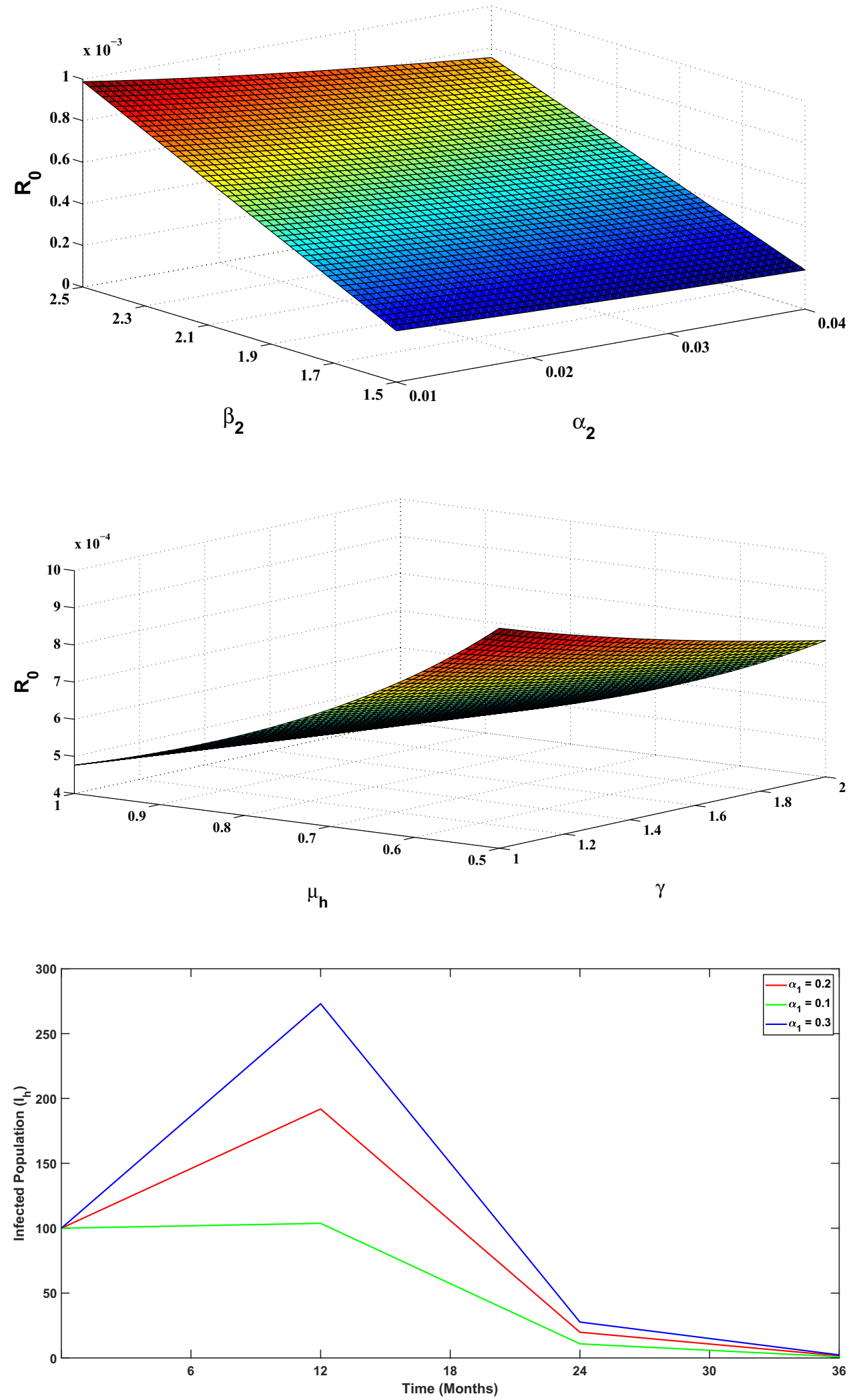

$\left(E_{\mathrm{r}}\right)$, susceptible $\left(S_{\mathrm{r}}\right)$ and infected rodents $\left(I_{\mathrm{r}}\right)$. Further, we have established the fundamental properties of the proposed model.
Basic reproduction number has been estimated using next-generation matrix technique. The proposed model exhibit two equilibrium points; disease free equilibrium 
Fig. 7 Variation in infected population over time for different values of $\delta_{\mathrm{h}}$; disease induced death rate of humans
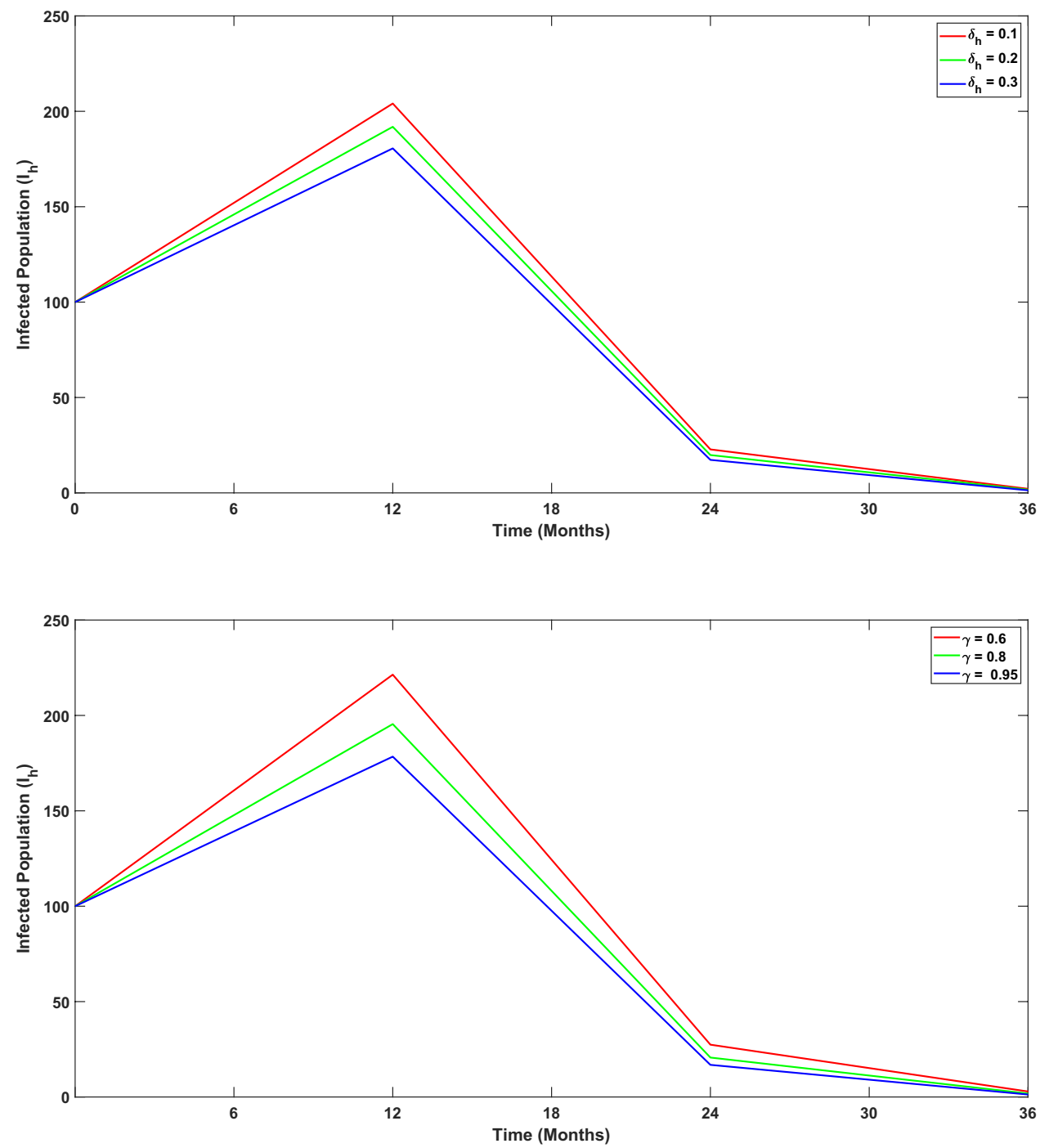

Fig. 8 Variation in infected population over time for different values of $\gamma$; recovery rate of humans

Fig. 9 Variation in infected population without any isolated interventions

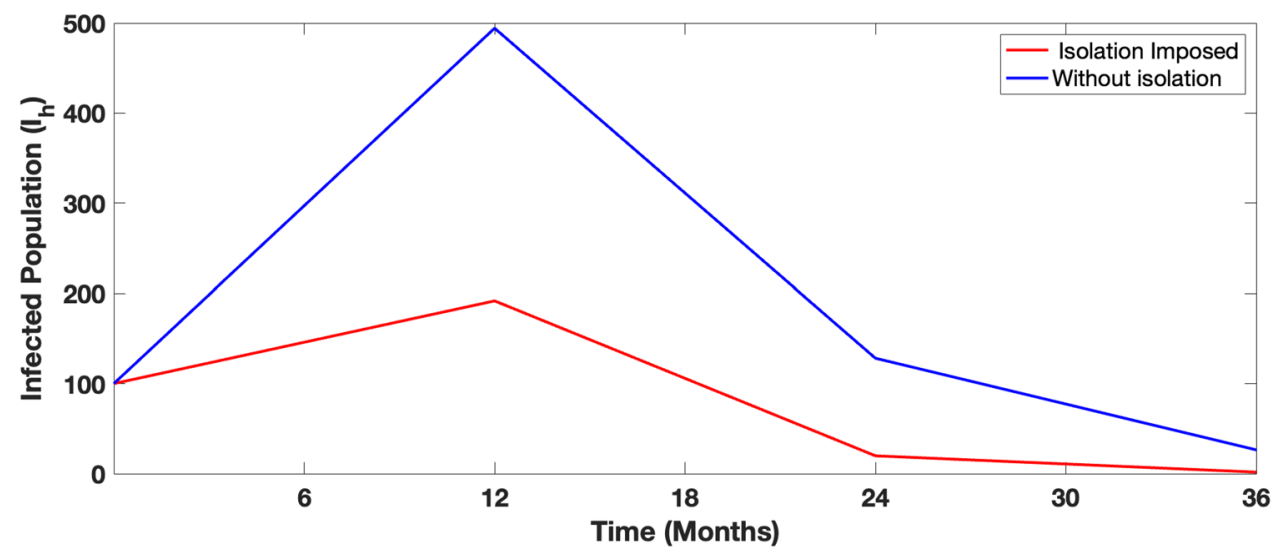

point and endemic equilibrium point. We have obtained the stability conditions for both of the equilibrium points. Further, the existence of the endemic equilibrium implies the possibility of the backward bifurcation. We have also derived the condition for the existence of the backward bifurcation. Further we have shown the sensitivity of various parameters involved in the model. The sensitivity index has been provided in Table 2. We found that $\alpha_{2}$, which is human to human contact rate is the most sensitive parameter in the transmission of the disease. Also, with the help of numerical 
simulations, we have shown the simultaneous effect of various parameters on the basic reproduction number $R_{0}$. Our analysis suggests that isolation of infected humans helps to reduce disease transmission. It is, therefore, realised from the simulation that isolation of the infected humans, is playing significant roles in the management and control of monkeypox virus.

\section{References}

Alakunle E, Moens U, Nchinda G, Okeke M (2020) Monkeypox virus in Nigeria: infection biology, epidemiology, and evolution. Viruses 12(11): 1257

Bankuru SV, Kossol S, Hou W, Mahmoudi P, Rychtář J, Taylor D (2020) A game-theoretic model of monkeypox to assess vaccination strategies. PeerJ 8:e9272

Bhunu C, Garira W, Magombedze G (2009) Mathematical analysis of a two strain hiv/aids model with antiretroviral treatment. Acta Biotheor 57(3):361-381

Bhunu C, Mushayabasa S (2011) Modelling the transmission dynamics of pox-like infections. IAENG Int J 41(2):1-9

Castillo-Chavez C, Song B (2004) Dynamical models of tuberculosis and their applications. Math Biosci Eng 1(2):361

CDC (2003) What you should know about monkeypox. https://www. cdc.gov/poxvirus/monkeypox/

Diekmann O, Heesterbeek J, Roberts MG (2010) The construction of next-generation matrices for compartmental epidemic models. J R Soc Interface 7(47):873-885

Durski KN, McCollum AM, Nakazawa Y, Petersen BW, Reynolds MG, Briand S, Djingarey MH, Olson V, Damon IK, Khalakdina A (2018) Emergence of monkeypox-west and central africa, 1970-2017. Morb Mortal Wkly Rep 67(10):306

Emeka P, Ounorah M, Eguda F, Babangida B (2020) Mathematical model for monkeypox virus transmission dynamics. Epidemiol Open Access 8(3): 1000348

Grant R, Nguyen L-BL, Breban R (2020) Modelling human-to-human transmission of monkeypox. Bull World Health Organ 98(9):638

Hutson CL, Gallardo-Romero N, Carroll DS, Clemmons C, Salzer JS, Nagy T, Hughes CM, Olson VA, Karem KL, Damon IK (2013) Transmissibility of the monkeypox virus clades via respiratory transmission: investigation using the prairie dog-monkeypox virus challenge system. PLoS ONE 8(2):e55488

Jezek Z, Szczeniowski M, Paluku K, Mutombo M, Grab B (1988) Human monkeypox: confusion with chickenpox. Acta Trop 45(4):297-307

Kalyan D, Reddy KG, Lakshminarayan K (2021) Sensitivity and elasticity analysis of novel corona virus transmission model: a mathematical approach. Sensors Int 2(1):100088
Kantele A, Chickering K, Vapalahti O, Rimoin A (2016) Emerging diseases-the monkeypox epidemic in the democratic republic of the congo. Clin Microbiol Infect 22(8):658-659

Kumar S, Sharma S, Singh F, Bhatnagar P, Kumari N (2021) A mathematical model for COVID-19 in Italy with possible control strategies. Mathematical analysis for transmission of COVID-19, p 101

Ladnyj I, Ziegler P, Kima E (1972) A human infection caused by monkeypox virus in basankusu territory, democratic Republic of the Congo. Bull World Health Organ 46(5):593

Meyer H, Ehmann R, Smith GL (2020) Smallpox in the post-eradication era. Viruses 12(2):138

Nguyen P, Ajisegiri W, Costantino V, Chughtai A, Maclntyre C (2021) Reemergence of human monkeypox and declining population immunity in the context of urbanization, Nigeria, 2017-2020. Emerg Infect Dis 27(4):1007-1014

Odom MR, Curtis Hendrickson R, Lefkowitz EJ (2009) Poxvirus protein evolution: family wide assessment of possible horizontal gene transfer events. Virus Res 144:233-249

Peter O, Viriyapong R, Oguntolu F, Yosyingyong P, Edogbanya H, MO A (2020) Stability and optimal control analysis of an scir epidemic model. J Math Comput Sci 2020(1):2722-2753

Rimoin AW, Mulembakani PM, Johnston SC, Smith JOL, Kisalu NK, Kinkela TL, Blumberg S, Thomassen HA, Pike BL, Fair JN et al (2010) Major increase in human monkeypox incidence 30 years after smallpox vaccination campaigns cease in the democratic republic of congo. Proc Natl Acad Sci 107(37):16262-16267

Samui P, Mondal J, Khajanchi S (2020) A mathematical model for COVID-19 transmission dynamics with a case study of India. Chaos Solit Fract 140:110173

Somma S, Akinwande N, Chado U (2019) A mathematical model of monkey pox virus transmission dynamics. IFE J Sci 21(1):195-204

TeWinkel RE (2019) Stability analysis for the equilibria of a monkeypox model. Thesis and Dissertations: University of Wisconsin. https://dc.uwm.edu/etd/2132

Usman S, Adamu II et al (2017) Modeling the transmission dynamics of the monkeypox virus infection with treatment and vaccination interventions. J Appl Math Phys 5(12):2335

van den Driessche P, Watmough J (2008) Further notes on the basic reproduction number. Springer, Berlin, Heidelberg, pp 159-178

Victorr Y, Hasifa N, Julius T (2020) Analysis of the model on the effect of seasonal factors on malaria transmission dynamics. $\mathbf{J}$ Appl Math 2020(4):19

Publisher's Note Springer Nature remains neutral with regard to jurisdictional claims in published maps and institutional affiliations. 